\title{
The Rheology of the Earth in the Intermediate Time Range
}

\author{
A. E. ScIIEIDEGGer $(*)$
}

Ricevuto il 5 Magagio 1970

\begin{abstract}
Summary. - The evidence bearing upon the rheology of the "tectonically significant layers" of the Larth ("tectonosphere") in the intermediate time range (4 hours to 15000 years) is analyzed. This evidence is based upon observations of rock-behavior in the laboratory, of seismic aftershock sequences, of Earth tides and of the decay of the Chandler wobble. It is shown that of the rheological models (Maxwell-material, Kelvin-material, and logarithmically creeping material) advocated in the literature, only that based on logarithmic creep does not contradict any of the observational evidence available to date. In addition, a strength limit may be present.
\end{abstract}

Riassunto. - Viene analizzata la validita dei risultati ottenuti dallo studio della reologia degli "strati tectonicamente significativi " della Terra (tectonosfera) in un intervallo intermedio di tempo (da 4 ore a 15000 anni). La prova di questa validità è basata sulle osservazioni del comportanento della roceia in laboratorio, su serie di repliche sismiche, sulle maree terrestri e sulla variazione della posizione dell'asse polare (Chandler wobble). Eे dimostrato ehe dei modelli reologici (Maxwell, Kelvin, deformazione logaritmica della materia) richiamati in Bibliografia, solo quello basato sulla deformazione loraritmica non è in contraddizione con la validità dei dati ottenuti dall'os. servazione. Si presenta inoltre l'ipotesi dell'esistenza di una fortezza limite (creep-slrenght) della quale ancora non si è potuto fare una determinazione.

\section{1. - INTRODUCTION.}

The scales of geodynamic phenomena reach spatially and temporally over a vast range. In the different time ranges, the response

(*) 201 Met. \& Min. Bldg., University of Illinois, Unbasa, Ill., (il801, U.S.A. 
of the material involved to the prevailing stresses is quite different. Some fourteen years ago, the present writer (21) summarized the then avalable knowledge of the rheologic behavior of the "tectonically significant" layers of the Earth ("tectonosphere"). The layers comprising the "tectonosphere" are the crust and a rather illdefined part of the upper mantle. The "rheological behavior of the tectonosphere" refers to its average behavior; conceivably the uppermost crust and the upper mantle could behave individually quite differently, but the scheme presented here refers to the combined effect of these possibly different individual responses. Since the earlier paper was written, much new evidence and observational data have been accumulated.

In the earlier paper, the time range of geodynamic phenomena, reaching from a fraction of a second (period of seismic waves) to hundreds of millions of years (continental drift) was divided into three intervals which were termed "short" (up to 4 lours), "intermediate" ( 4 hours to 15000 years) and "long" (longer than 15000 years).

It is the purpose of the present paper to review the new data pertaining to the "intermediate" time range and to interpret them in terms of their possible significance regarding the rheology of the tectonosphere for that time range. The provenance of the data is from direct measurements, from seismology, from tidal investigations, and from observations of wobbles in the Earth's rotation. In connection with this, it should be noted that the phenomena listed above may involve progressively larger portions of the Earth. Nevertheless, it is believed that all these effects concern mainly the upper parts of the Earth, and it will be seen that the rheological information that can be gleaned from them, all points in the same direction. I comparison with the earlier paper will show that two entirely new types of observations have been added; but with regard to those types which were taken into account earlier (viz., seismology and the wobble), entirely new data are now available whose interpretation will lead to a completely new assessment of the rheology of the Earth's tectonosphere in the intermediate time range. It will be seen that the commonly adopted Kelvin model for the description of the rheological behavior of the tectonosphere is inadequate. An alternate model, that of logarithmic creep, however, is compatible with all the new evidence. In the rheological discussion given here, the stresses refer to the stresses above a possible yield limit; it is difficult at this time to determine the possible existence and value of the latter. 


\section{2. - REYHEw ol: RHEOLOGY.}

The rheological equations of a body (sometimes also called equations of state) represent a relationship between the stress and strain tensors and their derivatives. The simplest relationships are linear ones. If the stresses depend linearly on the strain, one has an elastic material; if the stresses depend linearly on the strain rates, one has a viscous fluid. The standard rheological models that are then used to describe the behavior of different substances are materials showing various combinations of elastic and viscous behavior.

Let us specify this somewhat by focussing our attention on an isotropic, incompressible material. Then, only the ofl-diagonal elements of the varions tensors above a possible yield limit are of importance. This is the case which applies in creep and is sufficient for the discussion of what follows.

Thus, as noted above, a linear connection between shearing stress $\sigma$ (above a possible threshold) and shearing strain $2 \varepsilon$ ( $\varepsilon$ is the tensor component) leads to an elastic material. One usmally writes

$$
\sigma=2 \mu \varepsilon
$$

where $\mu$ is called the rigidity of the material. Similarly, one obtains a viscous fluid by setting

$$
\sigma=2 \eta \dot{\varepsilon}
$$

where $\eta$ is called the viscosity.

One then commonly considers two possible combinations of Fqs. [1] and [2]. The first is

$$
\dot{\varepsilon}=\frac{\dot{\sigma}}{2 \mu}+\frac{\sigma}{2 \eta}
$$

which represents a "Maxwell" fluid. Given a constant stress, the strain increases continuously linearly with time. The second possibility is

$$
\sigma=2 \mu \varepsilon+2 \eta \varepsilon
$$

which represents a "Kelvin-material". Given a constant stress $\sigma_{o}$, the strain adjusts itself exponentially to its final value $\sigma_{o} /(2 \mu)$ :

$$
\varepsilon=\frac{\sigma_{o}}{2 \mu}\left(1-e^{-\mu / \eta^{2}}\right)
$$


Of particular interest is the behavior of a Kelvin-material when oscillations occur. The equation of motion for such oscillations can be written symbolically

$$
\mu \varepsilon+\eta \dot{\varepsilon}=-\ddot{C \varepsilon} .
$$

If one makes the hypothesis that the oscillations are given by

$$
\varepsilon=\exp [(\alpha+i(v) t]
$$

one obtains

$$
\omega=\sqrt{\frac{\mu}{C}-\frac{\eta^{2}}{4 C^{2}}}
$$

and

$$
\alpha=-\eta /(2 C)
$$

showing that such oscillations will be damped. One can then eliminate the factor $C$ which yields a relation between the eigenfrequency (i) and the damping factor $\alpha$

$$
\alpha^{2}+\frac{2 \mu \alpha}{\eta}+\omega^{2}=0
$$

This allows one to estimate the ratio $\eta / \mu$ from measurements of $\omega$ and $a$. The presence of the damping in the Kelvin equation not only causes eigenoscillations to be damped, but causes also a phase-shift (a lag) in forced oscillations. The Kelvin equation of motion in this case may be written as follows

$$
\ddot{C \varepsilon}+\eta \dot{\varepsilon}+\mu \varepsilon=A \sin (1) t
$$

where $\eta$ is again the Kelvin viscosity, $\mu$ the rigidity, $C$ a constant of the system and $A \sin \omega t$ the disturbing force. The solution of the above equation is

$$
\varepsilon=\frac{A / C}{\sqrt{\left(\frac{\mu}{\zeta}--\omega^{2}\right)^{2}+\frac{\eta^{2}}{C^{2}} \omega^{2}}} \sin [\omega(t-\delta)]
$$

with

$$
\delta=\frac{1}{\omega} \arctan \frac{m \omega / C}{\mu / C-(1)^{2}}=\frac{1}{\omega} \arctan \frac{\eta \omega}{\mu-()^{2} C} .
$$

Noting that

$$
n=\sqrt{\mu / C}
$$


is the circular eigenfrequency of the undamped system, one can estimate the value of the Kelvin viscosity for the model in question. One again has to eliminate the unknown constant $C$

$$
C=\mu / n^{2}
$$

and has then

$$
\delta=\frac{1}{\omega} \arctan \frac{\eta \omega}{\mu-(1)^{2} \mu / n^{2}}=\frac{1}{\omega} \arctan \frac{n(\omega)}{\mu\left(1-\frac{\omega^{2}}{n^{2}}\right)}
$$

or

$$
\tau=\frac{\eta}{\mu}-\left(\frac{1}{(\prime)}-\frac{\omega}{n^{2}}\right) \tan \delta(\prime)
$$

For large $n$ (i.e., $n \gg \omega)$, this becomes $\left({ }^{9}\right)$

$$
\tau \sim \frac{1}{\omega} \tan \delta \omega
$$

The last equation enables one to get the ratio of the material constants $\eta / \mu$ from an observation of the phase-shift in forced oscillations.

The above rheological equations are all linear; they lead to exponential creep. However, the creep behavior of various materials, particularly of rock, is often not exponential, but logarithmic. In order to describe this case, Scheidegger ( ${ }^{23}$ ) has proposed the following rheological equation:

$$
\dot{\sigma}=2 \eta \ddot{\varepsilon}+\beta(\dot{\varepsilon}-C)^{2}
$$

where $\eta$ is the viscosity and $\beta$ a creep factor. For a constant stress $\sigma_{o}$, the strain becomes now a logarithmic function of time. One obtains upon integration of [16]

$$
\varepsilon=A+\frac{2 \eta}{\beta} \ln (1+B t)+C t
$$

or

$$
\varepsilon=\frac{2 \eta}{\hat{P}} \ln (a+b t)+C t
$$

where $A$ and $B$ (or $a$ and $b$ in the second form) are constants of integration. Furthermore, oscillations of such a material are no longer harmonic; their eigenfrequency depends on their amplitude. 
A special case of Eq. [17] is the so-called Lomnit\% (11) law which is valid for a constant acting stress $\sigma_{o}$

$$
\left[\varepsilon=\frac{\sigma_{o}}{\eta} 1+g \ln (1+B t) \mid\right.
$$

where $\mu$ is the rigidity. In the terminology of [17], this implies

$$
A=\frac{\sigma_{o}}{\mu} \quad \frac{2 \eta}{\beta}=q \frac{\sigma_{o}}{\mu} .
$$

Jeflreys $\left(^{5}\right)$ has taken this law as a stress-strain relation containing an explicit time dependence (rather than as a solution of [16] for particular boundary conditions), so that he writes, for variable stresses

$$
\varepsilon=\frac{1}{\mu} \int_{\tau=-\infty}^{t}\{1+q \log (1+B(t-\tau))\} d \sigma(\tau)
$$

However, in view of the fact that the stress-strain relation [16] is no longer linear, it does not seem entirely justified to apply the superposition principle implied in [18]. Nevertheless, if [17"] is taken as a stress-strain relation valid for variable stresses, Jeffreys $\left(^{5-7}\right)$ showed that the phase lag $\delta(\%$ induced in forced oscillations (cf. [9]) is approximately

$$
\delta \omega=\frac{1}{2} \pi q
$$

irrespective of frequency. It would, thus, be possible in principle to determine the constant $q$ from a measurement of a phase lag in an oscillatory system.

\section{3. - Direct OBservations.}

If one wishes to ascertain the rheological condition of the tectonosphere, one might first of all think of direct stress-strain measurements. Such measurements have been performed in the laboratory.

The difficulty in all such measurements is that the conditions obtaining in the tectonosphere are not easy to attain: whilst the timeelement in the "intermediate" range (weeks or years) does not present an unsurmountable obstacle, the pressure and temperature conditions can generally not be reproduced. Thus, with direct stress-strain 
measurements one las to confine himself to the conditions prevailing in the uppermost parts of the Earth's crust. This is less than the ideal situation, but still gives one an idea about what may be expected of the rheological behavior of certain rocks that make up the tectonosphere.

In this instance, we note that long ago Andrade (1) empirically analyzed the behavior of various materials under constant loading ( $\sigma=$ const) and came up with a general equation of the form

$$
\varepsilon=A+B E(t)+C t
$$

where $A, B$ and $C$ are constants and $E(t)$ is an empirical time-function.

In the above equation the term $C t$ is obviously characteristic of Maxwell-type creep; $C$ is zero in creep recovery.

The evidence regarding the time-function $E(t)$ was recently collected by Morlier (15). Accordingly, for some rocks one has

$$
E(t)=A\left(1-e^{-k t}\right)
$$

which corresponds to the behavior of a Kelvin-material. For rocks exhibiting this type of behavior, Morlier (15) [Table VI, p. 98] gives relaxation times of

$$
\tau=\frac{1}{k} \sim 1 \text { to } 16 \text { days }
$$

Using the ordinary short-term rigidity $\mu=2 \times 10^{12} \mathrm{cgs}$, this yelds a viscosity range of

$$
\eta \cong 1.7 \text { to } 27 \times 10^{17} \mathrm{cgs}
$$

Most rocks, however, do not have an exponential time-function, but a logarithmic one for a constant stress $\sigma_{o}$; a most common form is $\left({ }^{11}\right)$

$$
\varepsilon=\frac{\sigma}{\mu} 1+q \ln (1+B t)
$$

so that $A=\sigma_{o} / \mu ; B=q \sigma_{o} / \mu ; E(t)=\ln (1+B t)$; and $C=0$ in Eq. [20].

This can no longer be described in terms of linear models; one has to use in this case the nonlinear equation [16] derived by Scheidegger $\left.{ }^{23}\right)$. The behavior of various rocks has been analyzed in "terms of Eq. [17'] by Parsons and Hedley (17) [p. 332]. These authors assume in Eq. [17'] $a=0$, and obtain therewith values for the factor $2 \eta / \beta$ ranging from about $5.5 \times 10^{-6}$ for sandstone to about $6.7 \times 10^{-5}$ for potash. For potash, they require a Maxwell-term as well $\left(C=170 \times 10^{-6} \mathrm{hr}^{-1}\right.$, Eq. [17]), but this term is very small for 
sandstone $\left(C=4 \times 10^{-6} \mathrm{hr}^{-1}\right)$. At any rate, the lower of these limits seems to be more appropriate for an extrapolation to the Earth's crust rather than the higher one. The values given above may be taken as characteristic (in order of magnitude) for the "tectomosphere".

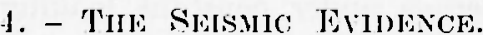

Seismology is concerned with the phenomena produced by earthquakes. As is well known (2:) these phenomena belong to two types: phenomena of wave propagation, and phenomena at the source. Much information has been accumulated on both these topics during the past len years. However, it should be noted that the information on wave propagation, including that on the free oscillations of the Earth, involves stress cycles whose prevailing periods are significantly below the "intermediate" time range.

Hence, the only important information for the "intermediate" time range to be gleaned from seismology is from the source-mechanisms, particularly from the time-erolution of aftershock series. Commonly, one has used the Beniofl-strain rebound model to explain themechanism of aftershocks. This model assumes that the main shock relieves the built-up stresses and that the subsequent aftershocks are the expression of the adjustment of a Kelvin-material to the stress-redistribution. Scheidegger ("1) has calculated the relaxation time $\tau$ required for this model from an analysis of aftershocks and obtained

$$
\tau \sim 2 \text { davs },
$$

leading to an estimate of the Kelvin viscosity, using the ordinary short-term rigidity $\mu=2 \times 10^{12}$ (gss, of $\eta=3 \times 10^{17}$ egs.

Inowever, the new information adduced over the last ten years showed that the Benioft model, and therefore the Kelvin model for the rheological behavior of the material in which the aftershocks take place, can no longer be maintained. In this instance, Ranalli and Scheidegger $\left({ }^{20}\right)$ made a careful analysis of a number of aftershock sequences and showed that aftershocks are the discontinuous manifestation of overall plastic creep by which the "tectonosphere" readjusts itself to the changing stress distributions. Accordingly, the material in the tectonically active layers of the Earth must be regarded (beyond the elastic limit) as a plastic substance exhibiting the phenomenon of creep as response to a stress-change. The details of the 
argument may be found in the cited paper of Ranalli and Scheidegger $\left({ }^{(20}\right)$ and need therefore not be repeated here.

Thus, we note that for an instantaneous stress change, the tectonosphere responds by creep. The aftershock-series corresponds to a recovery curve for macroscopic strain: this is the ratio of the total deformation divided by the total (not individual aftershock) earthquake volume.

Ranalli and Scheidegger $\left({ }^{20}\right)$ have shown that the (plastic macroscopic) strain $\varepsilon$ is proportional to the energ. stability, the strain rate is proportional to the aftershock frequency $n(t)$; the latter is given by Omori's law

$$
n(t)=u t^{\beta}
$$

where $\bar{\rho} \sim 1$, and estimates of the parameter $\alpha\left({ }^{19}\right)$ range from about 4 to 150 (dimensionless for $\beta=1$ ) for various aftershock sequences.

Thus, we have

$$
\dot{\varepsilon}(t)=\text { const } \cdot n(t)=\text { const } a t^{-1}
$$

and the strain rate curve (writing the constant as $l$ ) is griven by the integral of the expression above, leading to

$$
\varepsilon(t)=k \alpha \ln \varrho t
$$

which represents a form of logarithmic creep; $\underline{o}$ is a constant of integration.

The last strain-time equation corresponds again to the logarithmic areep equation [17'] with

$$
\begin{gathered}
\frac{2 \eta}{\beta}=\alpha k \\
a=0 \\
b=\varrho \\
c=0 .
\end{gathered}
$$

Unfortmately, no estimates of the ratio $2 \eta / \beta$ of the material constants can be made inasmuch as we see no way at the present time to estimate the proportionality constant $k$ between shearing strain rate and earthquake frequency. Nevertheless, the fact that logarithmic rather than exponential creep is found in the analysis of seismic aftershock sequences is in conformity with the findings from laboratory experiments with rock creep. 
In conclusion, it should perhaps be mentioned that Pshennikov $\left({ }^{18}\right)$ tried to fit a Maxwell-material to seismic aftershock sequences. However, his model fits the phenomenology of the aftershock process only very poorly and must be rejected [cf. the discussion by Ranalli and Scheidegger $\left.\left({ }^{20}\right)\right]$.

\section{5. - TIJES AND RILEOLOGY.}

The gravitational attraction of the Moon and the Sun upon the Earth produces deformations in the latter; these are commonly called "Earth tides". Because of the time-constants of the orbital motions of the Moon and the Earth, the periods of these tidal deformations fall into the "intermediate" time range, as defined in this paper.

In a first approximation, the tidal deformations of the solid Earth are elastic, implying an instantaneous proportionality between the surface-displacement (strain) and the tide-potential. This fact gives rise to the introduction of Love's and Shida's numbers expressing this proportionality $\left({ }^{13}\right)$ [p. 109]. However, it soon turned out that there is, in fact, no instantaneous proportionality between potential and displacement but generally a significant phase-lag (except for an insignificant advance in coastal Western Europe) of, in the semidiurnal wave, about $1.7^{\circ}$ in Central Europe and $3.5^{\circ}$ in the USSR, Asia and Japan $\left({ }^{14,16}\right)$; MacDonald $\left({ }^{12}\right)$ quotes an average of $2.16^{\circ}$ from astronomical observations. Such a phase-lag must cause energy dissipation in the Earth-Moon system, thereby affecting the evolution of the orbits in question $\left({ }^{2,8}\right)$.

The physical explanation of the observed phase-lag has been generally sought in a linear-type of anelasticity (13) [p. 350 ff.]. Indeed, in an oscillating system in which the damping is proportional to the velocity (strain rate), forced oscillations show a phase-lag with regard to the imposed oscillation force (cf. Sec. 2). From this phase-lag one can estimate the Kelvin viscosity of the tectonosphere. For the Earth, we have approximately (in hours)

$$
\begin{aligned}
n & =\frac{2 \pi}{1} \quad \begin{array}{l}
\text { (approximate lowest frequency mode of the free } \\
\text { oscillation } \left.\left({ }^{24}\right)\right)
\end{array} \\
\omega & =\frac{2 \pi}{6} \quad \text { (approximate frequency of the } M_{2} \text { tide) } \\
\delta \omega & =2.16^{\circ} ; \tan \delta \omega \sim 0.0378 .
\end{aligned}
$$


Thus the "relaxation time" $\tau=\eta / \mu$ becomes (cf. Eq. [14])

$$
\tau=\left(\frac{6}{2 \pi}-\frac{2 \pi}{6} \times \frac{1}{4 \pi^{2}}\right) \cdot 0.0378=0.0351 \mathrm{hrs} \simeq 126 \mathrm{sec} .
$$

This is a value that is very small. Using the ordinary short-term rigidity of

$$
\mu=2 \times 10^{12} \mathrm{cgs}
$$

yields for the Kelvin viscosity $\eta$

$$
\eta=2.5 \times 10^{14} \mathrm{cgs} .
$$

This is again a value which is very small. It does not agree with the values obtained from creep experiments of Kelvin-type rocks (see Sec. 2) nor does it agree with the value obtained from seismic aftershock sequences (see Sec. 3) if the latter are "forced" into a Kelvin model (Benioff strain rebound theory).

Since, in seismology, the Kelvin model has been shown to be inadequate in the "intermediate" time range (as evidenced by the decay of aftershock series) in any case, one will have to search for an explanation of tidal friction by models other than that of a Kelvin body.

A review of some such possibilities has been given by Iagus and Anderson $\left({ }^{10}\right)$. Accordingly, it may be expected that about one-half to two-thirds of the tidal energy dissipation may occur within the shallow seas. MacDonald (12) suggests that some of the dissipation of tidal energy in the solid parts of the Earth may be due to the grating of crustal blocks against each other. This idea comes rather close to an assumption of solid friction as a dissipative mechanism which, if it were assumed to occur in microscopic volumes rather than macroscopic blocks, would phenomenologically be that of logarithmic creep.

Thus, one concludes that the mechanism of energy dissipation in the Earth's tides is not yet very well understood. Assuming a Kelvin model requires viscosities which are much lower than those required by assuming the same model for other phenomena in the "intermediate" time range. Since the Kelvin model has been shown to be inadequate in other intermediate time range phenomena, it is probably best to abandon it in connection with the possible explanation of tidal dissipation as well. As an alternative possibility, friction in the shallow seas and logarithmic creep in the solid parts of the Earth could be invoked, and thus one might assume that a fundamental 
stress-strain relationship of the type of Eq. [16] is applicable in producing the phase-lag of the tides.

Indeed, the discussion of Jeffreys $\left(^{5}\right)$ of the origin of the phase-lag in a system with forced oscillations based on Isomnity' (11) law, can be applied to the tidal dissipation problem. Equation [19] leads to

$$
q=\frac{\partial(t)}{1 / 2 \pi}=\frac{2.16^{\circ}}{90^{\circ}}=0.024
$$

Unfortunately, this cammot be translated into terms of $n / 8$. since, for this purpose, $\sigma_{o}$ and $"$ would have to be known. Furthermore, Eq. [19] is based on a superposition principle whose validity, for an evidently nonlinear stress-strain law, is donbtful. The forced oscillations must be assumed to be no longer harmonic and thus the phase-lag is probably determined by the amplitude of the oscillations, not only by the material constants.

\section{6. - Rheology and tile Gitandler Wobble.}

The final effect concerning the Earth which we shall investigate regarding its implications upon the rheology of the tectonosphere is the Chandler wobble. This wobble $\left(^{4}\right)$ manifests itself by a periodic variation of the astronomically determined latitude of an observatory; the period is 430 days. It seems the variations become excited at irregular intervals; generally, it is now believed that the excitation is caused by the occurrence of large earthquakes (25). After excitation, the oscillations decay with a relaxation time (in amplitude) of some $10(5$ to 30$)$ periods. It stands to reason that some inferences can be made from the pattern of decay npon the rheology of the tectonosphere causing this decay.

In this vein, Scheidegger (21) has calculated the implications of the observed damping in terms of the Kelvin model. In this case, Equation [8] can immediately be used to make an estimate of the factor $\eta / \mu$ from a measurement of $\omega$ and $\alpha$. One obtains, with $T=$ $2 \pi / \omega=420$ days and $1 / \alpha=4200$ days,

$$
\tau=\frac{\eta}{\mu} \cong 2 \text { days } \sim 1.7 \times 10^{5} \mathrm{sec} .
$$

This is a value for the relaxation time $\tau$ which is not too different from that obtained by applying a Kelvin model to seismic aftershock series. 
However, the above is not the only interpretation possible for the origin of the decay of the Chmoller wobble. Thus, it has been tried to ascribe this decay to a Maxwell effect (3). Upon this basis, using a particular model of the structure of the Earth, Gerstenkorn ( $\left.{ }^{3}\right)$ found that the observed damping can be explained by assuming for the Maxwell-material in question

$$
\tau={ }_{\mu}^{\eta} \simeq 10^{8} \sec
$$

Using the ordinary short-term rigidity $\mu \sim 2 \times 10^{12} \mathrm{cgs}$, this yields a Maxwell viscosity of the earth of the order of $2 \times 10^{20}$ Poises. This is not too far from the order of mannitude of the very long-time viscosity deduced from such observations as isostatic adjustment (usually quoted as $\eta \sim 10^{22}$ to $10^{23}$ Poises).

Finally, some attempts have also been made to explain the damping of the Chandler wobble by the assumption of some logarithmic (reep law in the tectonosphere. Thus Jefrreys $\left(^{\circ}\right)$ used the form [17"] of Lomnitz' law and applied it to the damping in question. He obtained that this model would lead to a lag of $1 / 40$; using formula [19] this yields for $q$

$$
q=\frac{1}{40 \cdot 1 / 2 \pi}=\frac{1}{62.8}=0.016
$$

which is substantially less than the corresponding value obtained from an analysis of the Earth's tides. It does not agree with the damping values calculated from deep penetrating $\$$-waves, either, but in that case the time-range of the stress-ercles is much shorter so that, in our opinion, no agreement can be expected.

Nevertheless, the above discrepancies prompted Jeffreys to modify the logarithmic ereep law. However, we do not hold this to be necessary inasmuch as it is very doubtful that the superposition principle as used by Jefireys (Eq. [18]) is correct. If the logarithmice creep law is the solution, for particular boundary conditions, of some nonlinear stress-strain relation (cf. Eq. [16]), then the superposition of solutions emborlied in [18] is not admissible.

We therefore hold that it is probable that a solution for variable stresses of some nonlinear creep equation, corresponding to logarithmic creep under constant stress, will produce the required damping. Unfortunately, such calculations are not yet available. 


\section{7. - Evaluation.}

When we survey the discussion of the previous chapters, we note that basically three different rheological models have been advanced to describe the stress-strain behavior of the tectonosphere in the intermediate time range. These are: (a) Maxwell model, (b) Kelvin model and (c) the logarithmic creep model. The possible applicability of these models to the various phenomena under discussion here is set out in Table I.

An inspection of this Table I shows that the only interpretation of the rheological behavior of the tectonosphere in the intermediate time range that does not contradict some phenomenon, is that based on the logarithmic creep model.

To discuss the "score" for the individual models in detail, we may make the following remarks:

(a) The Maxwell model. It fits some of the direct laboratory measurements on rocks, but cannot be used for the explanation of seismic aftershock sequences. It does not seem to have been used in connection with the tides of the solid Earth, inasmuch as the response of the Earth to changing gravitational potentials is, in the first approximation, obviously elastic and not viscous. $A$ Maxwell-material, however, can be used for the treatment of the Chandler wobble.

(b) The Kelvin model. It fits the direct laboratory measurements on some rocks, but for most it is a poor model indeed. It cannot be used any longer for the explanation of the behavior of seismic aftershock sequences in the light of new evidence, although it used to be very popular in this context ("strain rebound theory"). It can be used in connection with the Chandler wobble and tidal friction, but the difference in the required material constants (e.g., $\tau$ ) in those two cases constitutes a severe difficulty. Thus, the Kelvin model must be rejected as a model of the rheological behavior of the tectonosphere in the intermediate time range.

(c) The logarithmic creep model. It fits all the phenomena discussed here, although in many instances not enough data are available as of yet to make a meaningful comparison with regard to the values of the material constants that are necessary to obtain a good fit between model and observations. Nevertheless, the logarithmic creep model seems to date to be the only one which must not be a priori rejected 
Table I

\begin{tabular}{|c|c|c|c|c|c|}
\hline Model & Phenomenon & Direct Measurements & $\begin{array}{c}\text { Seismic } \\
\text { Aftershocks }\end{array}$ & Tidal Friction & $\begin{array}{l}\text { Chandler } \\
\text { Wobble }\end{array}$ \\
\hline Maxwell & $\begin{array}{c}\text { constants } \\
\text { fit }\end{array}$ & $\begin{array}{c}C=4 \text { to } 170 \times 10^{-6} \mathrm{hr}^{-1} \\
\text { for some given stress } \\
\text { " ultimate creep" } \\
\text { seems to be of this type }\end{array}$ & $\begin{array}{l}\text { estimate not } \\
\text { possible } \\
\text { poor }\end{array}$ & not attempted & $\tau \cong 10^{8} \mathrm{sec}$ \\
\hline Kelvin & $\begin{array}{l}\text { constants } \\
\text { fit }\end{array}$ & $\begin{array}{c}\tau=1 \text { to } 16 \text { days } \\
\left(\eta \sim 1.7 \text { to } 27 \times 10^{17} \mathrm{cgs}\right) \\
\text { fits some rocks } \\
\text { but usually poor }\end{array}$ & $\begin{array}{c}\tau \cong 2 \text { days } \\
\left(\eta \sim 3 \times 10^{17} \mathrm{cgs}\right) \\
\text { poor }\end{array}$ & $\begin{array}{c}\tau \sim 126 \mathrm{sec} \\
\left(\eta \sim 2.5 \times 10^{14} \mathrm{cgs}\right)\end{array}$ & $\begin{array}{c}\tau \cong 2 \text { days } \\
\left(\eta \sim 3 \times 10^{17} \mathrm{cgs}\right. \\
\text { possible }\end{array}$ \\
\hline $\begin{array}{c}\text { logarithmic } \\
\text { creep }\end{array}$ & $\begin{array}{c}\text { constants } \\
\text { fit }\end{array}$ & $\begin{array}{c}\frac{2 \eta}{\hat{\rho}} \sim 5.5 \times 10^{-6} \text { to } 6.7 \times 10^{-5} \\
\operatorname{good}\end{array}$ & $\begin{array}{l}\text { estimate not } \\
\text { possible } \\
\text { good }\end{array}$ & $\begin{array}{c}q=0.024 \\
\text { possible }\end{array}$ & $\begin{array}{c}q=0.016 \\
\text { possible }\end{array}$ \\
\hline
\end{tabular}


for the explanation of the rheological behavior of the tectonosphere in the intermediate time range.

In all the above models, there may be a possible threshold stress below which they are not applicable. There is some evidence that such a stress-threshold (" creep-strength") exists, but no determination of it an be made at this time.

\section{ACKNOWLEDGMEN'.}

The writer wishes to thank Dr. Giorgio Ranalli of the Bedford Institute in Dartmonth, Nova Scotia, Canada, for reading and criticizing the manuseript of the present paper and for making pertinent suggestions for its improvements.

\section{REFERENCEA}

(1) Andrat) E. N. da C., On the viscous flow of metals and allied phenomena. "Proc. Roy. Soc." London A84, 1-12 (1910).

(2) Gerstenkorn II., On the controversy over the efiect of tidal friction upon the history of the earth-moon system. "Icarus", 7, 160-167 (1967).

(3) Gerstenkorn H., Damping of free nutation and relaxation time of the earth. "Icarus", 6, 292-297 (1967).

(") JeFFREYs H., Imperfections of elasticity in the small bodies of the solar system. "Monthl. Not. Roy. Astron. Soc." 117, 506-515 (1957).

$\left(^{5}\right)$ Jerrreys II., Roct creep, tidal friction and the moon's ellipticities. "MIonthl. Not. Roy. Astron. Soc." 118, 14-17 (1958).

(') Jefrreys II., Inelastie processes in the shell. "Geophys. J. Roy. Astr. Soc.", 14, 1-4 (1967).

(i) Jeffreys H., Crampin S., Rock ereep: a correction. "Monthl. Not. Roy. Astron. Soc.", 121, 571-577 (1960).

${ }^{8}$ ) KaULA W. M., Tidal dissipation by solid friction and the resulting orbilal evolution. "Revs. Geophys.", 2, 661-685 (1964).

(9) Knoporf L. Q., " Revs. Geophys.", 2, 625-660, (1964).

(10) Lagus P. L., Anderson D. L., Tidal dissipation in the earth and planets. "Phys. Earth. Planet. Int.", 1, 505-510 (1968).

(ii) Lomitz C., Creep measurements in igneous rock. "J. Geology", 64, 473-479 (1956).

(12) MacDonald G. J. F., Tidal friction. "Revs. Geophys.", 2, 467-541, (1964).

(13) Mrcomton P., The Earth Tides. London, Pergamon Press (1966). 
(1.4) Medcinor P., Oontribution apportée par les marees terrestres dans l'étude de la rotation de la terre in Continental Drift, ed. by Markowitz and Gninot. "Int. Astron. Un.", 71-76 (1968).

$\left.{ }^{15}\right)$ Morlier P., Le fluage des roches. "Ann. Inst. Tech. Bat. Trav. Publ.", 19, 89-112 (1966).

${ }^{(18)}$ PariǏskir N. N., Earth tides and the internal structure of the earth. "Bull. (Izv.) Acad. Sci. USSR. Geophys. Ser.", 1963, 193-215 (1963).

(17) Parsons R. C., Hedury D. G. F., The analysis of the viscous moperty of rocks jor classification. "Int. J. Rock Mech. Min. Sci.", 3, 325-335 (1966).

(18) Pshennikov K. V., Aflershock Mechanism and the Inelastic Properties of the Earth's Crust. "Nanka", Hoscow (1965).

(19) Ranalli G., A slatistical study of aftershock sequences. "Annali di Geofisica" XXII, 4, 359-397 (1969).

(20) Ravaldi G., Scinembgrier A. L., Rheology of the tectonosphere as inferred from seismic aftershock sequences. "Ann. Geolisica", XXII, 3, (1969).

(21) ScHeIDEgaen A. E., Rheology of the earth, the basic problem of geodynamics. "Canad. J. Phys.", 35, 383-397 (1957).

(22) ScheIdegGer A. E., Principles of Geodynamics, second ed. "Berlin Springer ", (1963).

${ }^{(23)}$ Scheidegger A. E., On the rheology of rock-creep. "Rock Mechanies" (1970).

(24) SurCirter C. B., Spherical oscillations of the earth. "Geophys. J. Roy. Astron. Soc.". 14, 171-177 (1967).

$\left.{ }^{(25}\right)$ Saryme D. E., Manshina L., Earthquakes and the observed motion of the rotation pole. "J. Geophys. Res.", 73, 7661-7673 (1968). 